the development of a biomarker-driven personalised medicine approach in IPF.

Objectives To improve our understanding of the pathogenesis of IPF by identifying clinically distinct groups of patients with IPF that could represent distinct disease endotypes.

Methods We systematically selected three publicly available datasets containing gene expression data measured from whole blood (220 IPF cases total). These datasets were co-normalised, pooled and clustered. We then compared clinical and demographic traits across clusters and used gene enrichment analysis to identify biological pathways and processes that were over-represented among the genes that were differentially expressed across clusters. A classifier was developed to assign additional individuals with IPF to a cluster using expression data from a minimal number of genes. We validated the classifier using three additional independent datasets (194 IPF cases total) and compared its performance at predicting survival in IPF to that of a previous transcriptomic prognostic biomarker for IPF.

Results We identified three clusters of IPF patients with distinct transcriptomic signatures. These clusters demonstrated statistically significant differences in lung function $(\mathrm{P}=0.009)$ and mortality $(\mathrm{P}=0.009)$ between groups. One cluster appeared to consist of patients with favourable lung function and survival over time (low risk cluster), whilst the other two clusters contained patients with worse lung function and reduced survival (high risk clusters). Gene enrichment analysis implicated dysregulation of mitochondrial homeostasis, apoptosis, cell cycle and innate and adaptive immunity in the pathogenesis of these groups. We developed and validated a 13-gene cluster classifier that predicted mortality in IPF (figure 1).

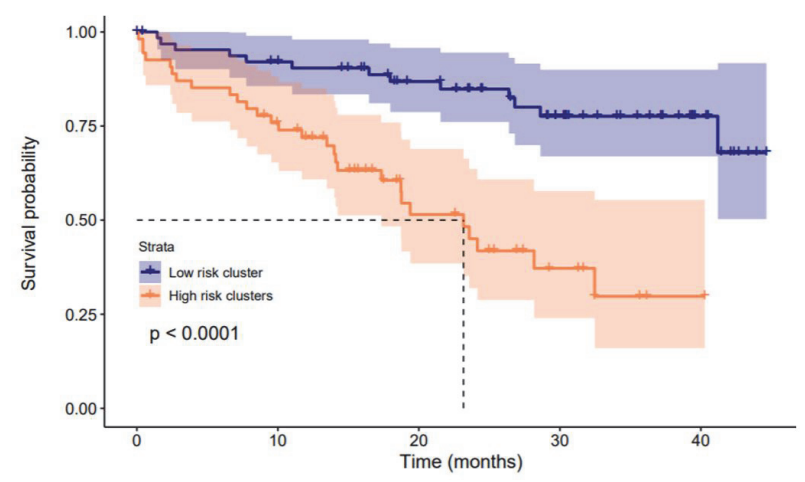

Abstract T2 Figure 1 Survival over time for the IPF subjects in the validation datasets, stratified by risk group according to our 13-gene classifier. The P-value on the plot is from a log-rank test testing the two curves for equality. The dashed line on the plot indicates the median survival time for the risk group if this could be calculated

Conclusions There are at least two groups of IPF patients with significant differences in survival and lung function that are discernible by blood gene expression signatures. These groups could be representative of distinct pathophysiological states, which would support the theory of multiple endotypes of IPF. Although more work must be done to confirm the existence of these endotypes, our classifier could be a useful tool in patient stratification and outcome prediction in IPF.

\section{T3 INTEGRATED TRANSCRIPTOMIC ANALYSIS OF HUMAN TUBERCULOSIS GRANULOMAS AND A BIOMIMETIC MODEL IDENTIFIES SPHINGOSINE KINASE 1 AS A POTENTIAL THERAPEUTIC TARGET}

${ }^{1} M T$ Reichmann, 'LB Tezera, ${ }^{1}$ AF Vallejo, ${ }^{2} M$ Vukmirovic, ${ }^{3} R$ Xiao, 'S Jogai, 'S Wilson, ${ }^{1} \mathrm{BG}$ Marshall, ${ }^{1} \mathrm{MG}$ Jones, ${ }^{4} \mathrm{~A}$ Leslie, ${ }^{3} \mathrm{~J}$ D'Armiento, ${ }^{2} \mathrm{~N}$ Kaminski, ${ }^{1} \mathrm{ME}$ Polak, ${ }^{1} \mathrm{PT}$ Elkington. ${ }^{1}$ University of Southampton, Southampton, UK; ${ }^{2}$ Yale School of Medicine, New Haven, USA; ${ }^{3}$ Columbia University Medical Center, New York, USA; ${ }^{4}$ Africa Health Research Institute, KwaZulu Natal, South Africa

\subsection{6/thorax-2021-BTSabstracts.3}

The global burden of tuberculosis (TB) continues at pandemic proportions, currently with a quarter of the world's population infected with Mycobacterium tuberculosis (Mtb), and 1.4 million people dying from TB in 2019 (WHO, 2020). Mtb has undergone prolonged co-evolution with humans, with the balance between protective and pathological host responses likely to play a key role in determining clinical severity. Sarcoidosis is another granulomatous condition primarily affecting the lung and lymph nodes. TB and sarcoidosis share histological and clinical features, which can be indistinguishable, including immune-related phenomena which suggest shared immunological processes. Despite scientific advances, the immunopathology of TB and sarcoidosis remain incompletely understood. Here, we show the combination of unbiased analysis of patient samples and a biomimetic model has established a translational pipeline to identify new therapeutic approaches.

We hypothesised that whole transcriptome analysis of human TB granulomas isolated by laser capture microdissection could identify therapeutic targets, and that comparison with sarcoidosis would identify disease-specific mechanisms.

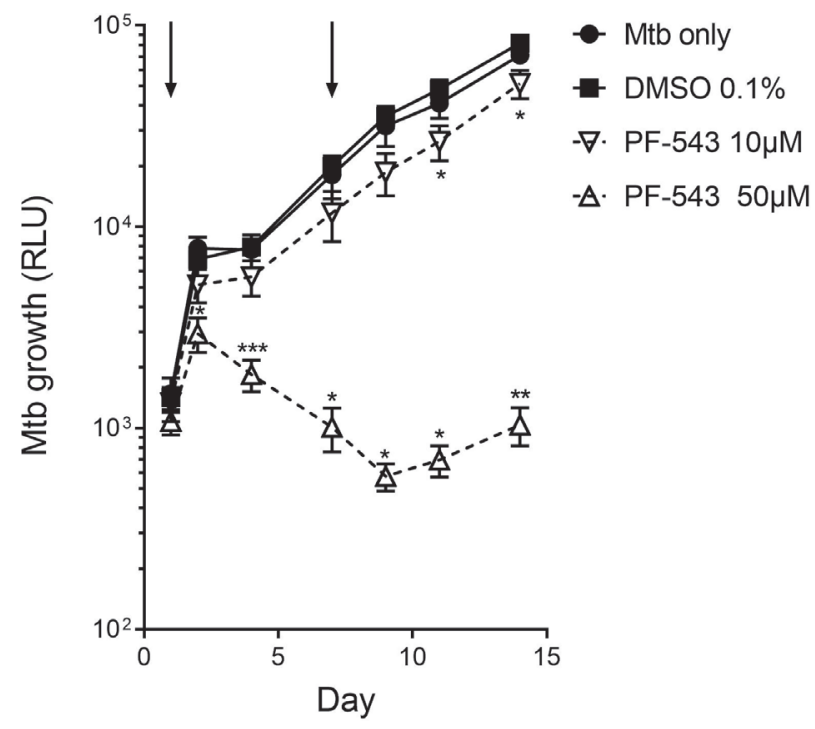

Abstract T3 Figure 1 Mtb growth detected in 3D collagen model measured by luminescence (RLU, relative light units): untreated (black circles), DMSO (black squares), and SphK1 inhibitor PF-543 (unfilled triangles). Black arrows: drug addition on days 1 and 7. Experiments were performed on at least five occasions using PBMCs from five separate healthy donors with triplicate conditions. Data presented are from a representative donor and are consistent across donors. Analysis: two way ANOVA with Dunnett's Multiple Comparison Test, error bars: SD.

${ }^{*} p<0.05,{ }^{* *} p<0.01,{ }^{* * *} p<0.001$ 
Treatment-naïve biopsies were analysed from seven TB patients, ten sarcoidosis patients and seven control samples. Bioinformatic analysis of RNAseq data identified one third of differentially expressed genes were communal to TB and sarcoidosis relative to control samples (absolute $\log _{2}$ fold change $\geq 1.5$, adjusted $\mathrm{P}$ value $<0.05$ ), with overlap of numerous pathways, including the extracellular matrix and cytokine signalling. Importantly, a TB unique cluster demonstrates TB results from a dysregulated inflammatory immune response, whereas a sarcoidosis predominant cluster relates to elevated lysosomal activity.

To translate these insights, we compared three primary human cell culture models: classical 2D; 3D alginate; and 3D collagen model. We demonstrated the Mtb-infected 3D collagen model most closely reflected clinical TB biopsies. We investigated signalling pathways shared between human disease and the 3D model, and used a systematic selection process to identify twelve intracellular enzymes as potential therapeutic targets. Sphingosine kinase 1 (SphK1) inhibition controlled $\mathrm{Mtb}$ growth in a dose-dependent manner, concurrently lowering intracellular $\mathrm{pH}$ in infected monocytes and suppressing inflammatory mediator secretion. Immunohistochemical staining confirmed that SphK1 is expressed in human lung TB granulomas, and therefore represents a potential novel hostdirected therapeutic target to improve TB outcomes.

\section{T4 REINFECTION WITH INFLUENZA A VIRUS LEADS TO RAPID CHANGES IN IMMUNOMODULATORY MOLECULES AND INFLAMMATORY SUBTYPES OF LUNG FIBROBLASTS AND EPITHELIAL CELLS}

${ }^{1} \mathrm{JC}$ Worrell, ${ }^{1} \mathrm{G}$ Finney, ${ }^{1} \mathrm{KE}$ Hargrave, ${ }^{1} \mathrm{C}$ Hansell, ${ }^{2} \mathrm{~J}$ Singh Nijjar, ${ }^{1} \mathrm{~F}$ Morton, ${ }^{1} \mathrm{~J} \mathrm{Cole}$, ${ }^{1}$ MKL MacLeod. 'Institute of Infection, Immunity and Inflammation, University of Glasgow, Glasgow, UK; ${ }^{2}$ Department of Medicine, University of Cambridge, Cambridge, UK

\subsection{6/thorax-2021-BTSabstracts.4}

Introduction and Objectives Influenza A virus (IAV) induces respiratory infections resulting in significant global mortality. Stromal cells are essential sources of chemokines and growth factors that promote immune cell survival and anti-viral responses. The concept that stromal cells are permanently altered by inflammatory responses is referred to as trained immunity. We hypothesise that IAV infections influence future immune responses via changes to lung stromal cells, enabling rapid communication with immune cells following subsequent infection.

Methods C57BL/6 mice were infected intranasally with IAV (WSN, 150PFU) for 30 days and subsequently re-challenged with IAV (X31, 200PFU) for 2 days. Mice were sacrificed at day $0,2,30$ and 32 post infection. A Nanostring assay was used to examine the transcriptional profiles of FACS sorted lung fibroblasts and epithelial cells. Differentially expressed genes were validated by qPCR and flow cytometry. The expression of upstream transcriptional drivers of these changes were compared with publicly available RNA-seq and ChIP-seq datasets. The potential impact of these changes on stromalimmune cell communication were assessed using immunohistochemistry and immunofluorescence.

Results Genes involved in $\mathrm{T}$ cell communication were significantly upregulated in lung fibroblasts (Cd274, Cxcl10) and epithelial cells (Tnfsf10, Icam2) following secondary IAV infection (d32), compared to $\mathrm{d} 30$. These changes were accompanied by elevated IFN-response genes (Bst2, Ifi47, Irf7).
Fibroblasts displayed enrichment in genes involved in biological processes regulating $\mathrm{T}$ cell activation, while epithelial cells were enriched for genes that regulate cytokines (IFN $\alpha / \beta$, $\mathrm{TNF} \alpha$ ). After secondary IAV infection, stromal cells rapidly upregulated genes involved in antigen processing and presentation (Tap1, Tapbp), compared to $\mathrm{d} 30$. The transcription factor SpiB was identified as a shared upstream regulator of these genes. Interestingly, in contrast to $\mathrm{d} 2$ post primary infection, MHCII+ epithelial cells were elevated following secondary infection, while the frequency of IFN-responsive fibroblasts was decreased. $\mathrm{T}$ and $\mathrm{B}$ cells were located near airway epithelial cells at $\mathrm{d} 30$ and were retained following secondary infection, indicating ongoing communication between these cells.

Conclusions Our data, show that lung fibroblasts and epithelial cells can display overlapping and functionally discrete responses to IAV infection, enabling them to rapidly communicate with lung $\mathrm{T}$ cells following a subsequent infection.

Please refer to page A188 for declarations of interest related to this abstract.

\section{T5 RESPIRATORY PARTICLE AND DROPLET EMISSION DURING SPEECH AND EXERCISE}

${ }^{1,2,3} \mathrm{CM}$ Orton, ${ }^{4} \mathrm{HE}$ Symons, ${ }^{1} \mathrm{~B}$ Moseley, ${ }^{4} \mathrm{~J}$ Archer, ${ }^{5} \mathrm{NA}$ Watson, ${ }^{1,3} \mathrm{KEJ}$ Philip, ${ }^{6} \mathrm{~B}$ SaccenteKennedy, ${ }^{7}$ DAE Costello, ${ }^{8} \mathrm{WJ}$ Browne, ${ }^{9,10} \mathrm{JD}$ Calder, ${ }^{4} \mathrm{BR}$ Bzdek, ${ }^{1,11} \mathrm{JH}$ Hull, ${ }^{4} \mathrm{JP}$ Reid,

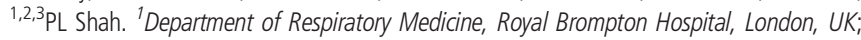
${ }^{2}$ Department of Respiratory Medicine, Chelsea and Westminster Hospital, London, UK; ${ }^{3}$ National Heart and Lung Institute, Imperial College London, London, UK; ${ }^{4}$ School of Chemistry, University of Bristol, Bristol, UK; ${ }^{5}$ Department of Ear, Nose and Throat Surgery, Guys and St. Thomas NHS Foundation Trust, London, UK; ${ }^{6}$ Speech and Language Therapy Department, Royal National Ear Nose and Throat Hospital, London, UK; ${ }^{\top}$ Ear, Nose and Throat Department, Wexham Park Hospital, Slough, UK; ${ }^{8}$ School of Education, University of Bristol, Bristol, UK; ${ }^{9}$ Department of Bioengineering, Imperial College London, London, UKi ${ }^{10}$ Fortius Clinic, London, UK; ${ }^{11}$ Institute of Sport, Exercise and Health (ISEH), UCL, London, UK

\subsection{6/thorax-2021-BTSabstracts.5}

Background The coronavirus disease-19 (COVID-19) pandemic has profoundly impacted sports and exercise, disrupting a plethora of events worldwide. Aerosol transmission is increasingly recognised as an important route for severe acute respiratory syndrome coronavirus 2 (SARS-CoV-2), with systematic evaluation of particulate matter release during exercise required to understand and mitigate transmission risk.

Methods Healthy participants $(n=25)$ performed a two stepped, flat-wave cardiopulmonary exercise test (CPET) on a cycle ergometer to replicate vigorous exercise (80\% of anaerobic workload) and high intensity exercise (anaerobic workload $+30 \%$ of the difference between anaerobic workload and peak workload), as determined by a maximally exhaustive CPET performed one hour previously. Concurrent measurements of aerosol and ventilatory data were recorded via a sampling line connected to an aerodynamic particle sizer (APS). Further synchronous data were collected at rest and when speaking at 70-80dBA. Droplet data were collected at rest and during high intensity exercise, using water sensitive paper.

Findings Median aerosol number concentration during speaking (at $70-80 \mathrm{dBA}), 0 \cdot 26 \mathrm{~cm}^{-3}$, was greater than during vigorous exercise, $0.12 \mathrm{~cm}^{-3}(\mathrm{p}<0.001)$ but not different to high intensity exercise, $0.24 \mathrm{~cm}^{-3}(\mathrm{p}=0 \cdot 92)$. Median aerosol mass concentration during speaking, $0.40 \mu \mathrm{g} / \mathrm{m}^{-3}$, was greater than during vigorous exercise $\left(0 \cdot 17 \mu \mathrm{g} / \mathrm{m}^{-3}, \mathrm{p}<0 \cdot 001\right)$, but not different to 